\title{
El rol de las fuentes en las noticias
}

\author{
Nicolás Rotelli \\ Universidad de Ciencias Empresariales y Sociales, Argentina \\ nicolasrotelli@gmail.com
}

Recibido: 6/3/2018 / Aceptado: 4/4/2018

doi: 10.26439/contratexto2018.n029.1818

\begin{abstract}
RESUMEN. Este artículo busca identificar y analizar la presencia de las fuentes informativas en las noticias publicadas en la prensa gráfica argentina. Conocer la acción de las fuentes es importante para una mejor comprensión del proceso de construcción de la información que circula en los medios, ya que una parte significativa del contenido es producida con la intervención de las relaciones públicas. Por ello, el artículo se propone identificar y explicar el rol de las fuentes institucionales, analizando la presencia de este tipo de fuentes en las noticias con relación a dos variables: la temática a la que se refieren las noticias y los medios en que estas fueron publicadas. Esto tiene el objetivo de observar si la presencia de la fuente permanece estable o se modifica cuando cambia la temática de la noticia o el medio.
\end{abstract}

Palabras clave: fuentes / periodismo / relaciones públicas / noticias / medios de comunicación 


\section{The Role of Sources in the News}

ABSTRACT. This paper aims to identify and analyze the presence of informative sources in news published in the Argentine graphic press. It is important to know how sources perform for a better understanding of the building process of the information circulating in the media, because a significant part of the news content is produced with the intervention of public relations. Therefore, this paper intends to identify and explain the role of institutional sources, by analyzing the presence of this type of sources in the news in relation to two variables: the subject to which the news refer and the media in which the news were published. Its objective is to observe whether the presence of the source remains stable or is modified when the subject of the news or the media change. Keywords: sources / journalism / public relations / news / media 


\section{Introducción: las fuentes en el proceso de construcción de la información}

$\mathrm{T}$ oda noticia tiene su punto de origen en una fuente. La fuente informativa es "todo aquel o aquello que suministra información con relación a los hechos y sucesos que son objeto de la noticia" (Ortiz, en Ambort, Loyola, Ortiz y Tobar, 1996, p. 130). Las instituciones y actores sociales que quieren llevar su voz al público a través de los medios de comunicación buscan constituirse en fuentes institucionales confiables para los medios (Duarte, 2011). Como señala Gomis (1991), las instituciones que esperan obtener un rédito con la publicación de una información son las que inician la difusión de un acontecimiento (p. 64). Así, una de las herramientas tácticas de la comunicación organizacional es la gestión de las relaciones con la prensa.

En este artículo, se considerarán como fuentes a aquellas personas o instituciones que se consignan expresamente como informantes de los datos o declaraciones que aparecen en las noticias. Se buscará identificar y analizar la presencia de las fuentes informativas en las noticias, asociando su aparición con dos variables: las temáticas de las noticias y los medios en que se publicaron.

Las fuentes informativas y los periodistas son dos actores centrales en el proceso de construcción de las noticias. McCombs (2006) señala que los periodistas solo pueden observar una pequeña fracción de las situaciones y hechos que se producen cotidianamente (p. 197). Por ello, la función que cumplen las fuentes informativas es clave porque brindan de manera rutinaria a los periodistas el material para que elaboren las noticias. De allí la importancia que tienen los profesionales de las oficinas o gabinetes de prensa y los funcionarios relacionados con la información pública en el proceso de construcción de las noticias.

Cada vez es más relevante el rol que desempeñan las fuentes institucionales en el proceso de construcción de las noticias, al proveer al periodista de datos y declaraciones que muchas veces se transforman en el contenido principal de las historias publicadas. Hoy, las oficinas responsables de las relaciones con la prensa cuentan con profesionales formados y equipamiento técnico a la altura de los grandes medios de comunicación (Amado, 2010, 2016b). Diversas investigaciones comprobaron la efectividad de las relaciones públicas como fuente y explican este fenómeno por una combinación de factores, entre los que destacan el ahorro económico que genera en los medios el no tener que producir información propia y la habituación a canales de rutina que permiten a los periodistas obtener información en tiempo real (McQuail, 1998, p. 194).

Los antecedentes sobre el estudio del establecimiento de la agenda de los medios (McCombs, 2006), así como la correlación entre la agenda de temas impulsada por las organizaciones $y$ las noticias publicadas, nos llevan a pensar en la necesidad de continuar 
investigando sobre este tema, aportando datos actuales sobre el rol de las fuentes institucionales en el proceso de producción de las noticias. Las fuentes de los poderes políticos y económicos tienen más posibilidades de llevar su voz a las noticias (McQuail, 1998; Castells, 2009) y se convierten, con frecuencia, en los actores más consultados por el periodismo. A su vez, voces que serían importantes para la opinión pública se quedan en varias ocasiones sin llegar a las páginas de los diarios por carecer de recursos (McQuail, 1998, p. 131). El estatus y la capacidad de producción de la fuente son dos elementos centrales para explicar el éxito de algunos actores en lograr que su visión de los hechos sea publicada.

En este trabajo, se parte de la premisa que considera a la noticia como un proceso del que participan diversos actores: los periodistas, las fuentes informativas y el público (Martini, 2000). Se analizará aquí la relación entre dos de ellos: las fuentes y los periodistas. La perspectiva teórica adoptada para el análisis de los procesos de producción de noticias es la del newsmaking (Martini y Luchessi, 2004): los procedimientos de trabajo que normalizan los procesos y facilitan las decisiones periodísticas se conocen como rutinas productivas dentro de la teoría de la noticia. Esta investigación considera fundamental analizar el proceso de producción de noticias que circulan en los medios atendiendo a lo que efectivamente producen los periodistas (Hanitzsch y Mellado, 2011; Mellado y Van Dalen, 2013).
Además, desde los estudios de las prácticas profesionales del periodismo, se establecen cuáles son los factores de influencia en el proceso de producción de las noticias, dentro de los cuales se encuentra la acción de las fuentes (Mellado y Van Dalen, 2013; Mellado, 2015). Así, el enfoque aquí adoptado se basa en la constatación de que los Estados, empresas y organizaciones de la sociedad civil han ido perfeccionando técnicas de comunicación que les permiten participar activamente en el sistema de medios con los que interactúan en un proceso de influencia recíproca (Amado, 2014). Un elemento insoslayable en la construcción de la información pública es la profesionalización en las herramientas y tácticas de comunicación de diversos actores sociales. Este proceso se ha acelerado en las dos últimas décadas con medios que carecen de recursos para la producción propia de información, mientras que las organizaciones, en especial las más poderosas, fueron asignando cada vez mayores recursos técnicos y económicos para la gestión de las comunicaciones institucionales, recursos que muchas veces atraen a profesionales que antes trabajaban en las redacciones (Amado, 2016a).

\section{Metodología: investigar la presencia de las fuentes en las noticias publicadas}

En este artículo, se retoman los datos obtenidos en el capítulo argentino del estudio internacional Journalistic 
Role Performance ${ }^{1}$, donde se relevaron, mediante la técnica de análisis de contenido, 3400 noticias de 4 diarios de temática nacional desde el 2 de enero del 2012 al 31 de diciembre del 2013. Se seleccionaron los diarios Clarín, Diario Popular, Página 12 y La Nación. La elección de estos medios obedece, por un lado, a que son los periódicos de temática nacional de mayor tirada y, además, a que tienen diferentes públicos $\mathrm{y}$ adscripción ideológica, lo que nos permitirá observar regularidades en las relaciones entre las fuentes y los periodistas, más allá del medio donde se publique la noticia analizada. Podemos establecer ciertos rasgos identitarios de cada diario analizado: en cuanto a la orientación ideológica, Clarín y Diario Popular son de centro, mientras que $\mathrm{La}$ Nación es de centroderecha y Página 12 de centroizquierda; en lo que respecta a la orientación de mercado, La Nación y Página 12 tienen como público objetivo a las élites locales, mientras que Clarín y Diario Popular intentan obtener el segmento popular del mercado.

Para conseguir una muestra sistemática de noticias que fuera representativa de cada año estudiado, se analizaron las ediciones de una semana para cada semestre, armadas con el método de semana construida ${ }^{2}$ (Riffe, Aust y Lacy, 1993). Este método consiste en seleccionar un día de diferentes semanas elegidas con intervalos aleatorios para componer una muestra que forme una "semana completa", de modo que todos los días de la semana y los meses del año estén representados.

Se consideró como unidad de análisis a cada noticia, definida como el grupo de elementos verbales y gráficos que se refieren a un mismo tema, dentro de las secciones de actualidad de cada diario. Noticias incluidas en revistas, suplementos o ediciones especiales de los diarios no se tuvieron en cuenta para la muestra. Como el interés de la investigación estuvo enfocado en las prácticas periodísticas de construcción de la información, quedaron excluidos las notas de opinión y los editoriales de los diarios. Por la misma razón, también se prescindió de publicidades, pronósticos del tiempo, chistes, sección de juegos, horóscopo, programación de TV o de otros espectáculos deportivos o culturales. Tampoco se incluyeron en la muestra las entrevistas publicadas cuando estas constituían el $50 \%$ o más de la noticia. Fueron analizadas 1247 noticias del diario Clarín, 802 de Página 12, 682 de $L a$ Nación y 669 de Diario Popular.

1 La red está conformada por investigadores de Alemania, Brasil, Argentina, Chile, China, Cuba, Chipre, Ecuador, España, Estados Unidos, Filipinas, Grecia, Hong Kong, Hungría, India, Italia, Irlanda, Malasia, México, Polonia, Rusia, Suiza y Tailandia. Está disponible en http://www. journalisticperformance.org/

2 El método de la semana construida consiste en un muestreo estratificado sistemático (Riffe, Aust y Lacy, 1993). 
Con relación a los objetivos de este artículo, mencionados anteriormente, se trabajó tres preguntas de investigación sobre el rol de las fuentes en las noticias:

P1: Las fuentes informativas institucionales tienen una presencia significativa en las noticias.

P2: La presencia de fuentes informativas varía significativamente en función de las temáticas de las noticias.

P3: La presencia de fuentes informativas varía significativamente en función de los medios en los que se publican las noticias.

Estas preguntas buscan complementar, mediante el foco puesto en el análisis de las fuentes informativas, las observaciones registradas por diversas investigaciones sobre la práctica periodística en América Latina, realizadas en el marco del estudio JRP (Mellado y Van Dalen, 2013; Hellmueller y Mellado, 2016; Mellado et al., 2016; Mellado et al., 2017).

\section{Las fuentes informativas en relación con las temáticas y los medios de comunicación}

Se codificó cada fuente que el periodista consultaba, independientemente de las veces que aparecía citada en la noticia. De esta manera, se consignó cada ocasión que un tipo de fuente aparecía por lo menos una vez en la noticia. Además, se identificó cuál de todas era la fuente principal de cada nota, a través del registro de aquella que recibió el mayor espacio de información o el mayor número de referencias.
La ausencia de fuentes en la noticia también fue un indicador para tener en cuenta.

Un primer punto de análisis fue identificar a los actores sociales que se convierten en fuentes para los periodistas. Se definieron los siguientes tipos de fuentes:

- Fuente estatal o partido político: funcionarios públicos de los poderes ejecutivo y legislativo, miembros de partidos políticos.

- Fuente sector empresarial: voceros y representantes del sector empresarial.

- Fuente sociedad civil: miembros de la sociedad civil organizada.

- Fuente ciudadanos comunes: personas que hablan a título personal, sin representar a ninguna organización.

- Fuente medios: información provista por medios de comunicación.

- Fuente expertos: especialistas de un área del conocimiento, académicos e investigadores.

- Fuente anónima: fuenteno identificada.

- Otras fuentes.

Al analizar los diversos tipos de fuentes noticiosas, observamos la clara preeminencia de las estatales y políticas por encima del resto de los actores sociales: el $35 \%$ de las noticias tienen una o más fuentes estatales. El segundo tipo de fuente con mayor presencia son las anónimas, es decir, aquellas que no están identificadas plenamente, presentes en el $13 \%$ de las noticias. También 
con el $13 \%$ aparecen las fuentes de la sociedad civil organizada: sindicatos, iglesias y organizaciones del tercer sector. Luego, con una presencia del $11 \%$, se encuentran la ciudadanía no organizada y los medios de comunicación. Finalmente, los expertos y las fuentes del sector empresarial son citados en un $7 \%$ de las noticias. Aquí podemos observar el primer indicador de la presencia de las fuentes institucionales en las noticias, ya que solo 1 de cada 10 incluye como fuente a actores no organizados.

Ahora bien, cuando analizamos cuál es la fuente principal de la noticia, estos datos se mantienen. Personas o instituciones estatales y políticas son la fuente principal en más del $34 \%$ de las noticias analizadas. Organizaciones de la sociedad civil son la fuente principal en el $10 \%$ de las noticias, seguidas por ciudadanos comunes. El porcentaje de las noticias donde la fuente principal no aparece plenamente identificada no es despreciable: el $8 \%$. Otro dato importante es que casi un $19 \%$ de las noticias no tienen fuente. Los expertos en diferentes temáticas, como salud, educación, transporte o energía, son la fuente principal en menos de 1 de cada 10 noticias. Esto nos lleva a pensar en la necesidad de incluir voces que permitan al lector contar con mayores datos y perspectivas idóneas para entender las noticias en contextos complejos como el actual.

Cuando analizamos las fuentes por temáticas de las noticias (tabla 1), observamos, por un lado, la ubicuidad de las estatales y políticas en toda la agenda de temas. Esto es especialmente importante en temas de gobierno, campañas políticas, policiales y economía, donde es previsible que la fuente oficial del Estado tenga un rol preponderante. Pero este rol se traslada a otros temas, tales como energía, transportes y protestas, en donde las fuentes estatales siguen siendo claramente dominantes en cuanto a niveles de presencia. En temas de gobierno es significativo el uso de fuentes anónimas, un dato importante, ya que son temas de interés público sobre los cuales sería deseable tener la voz de fuentes plenamente identificadas.

En temas policiales se observa una importante presencia de fuentes anónimas. Muchas veces los testigos del caso sobre el que se escribe no quieren dar a conocer su identidad. Otras veces es la misma fuente gubernamental, en este caso la policía, la que no aparece identificada plenamente, por cuestiones procedimentales o de protección. En esta temática, los ciudadanos no organizados son una fuente de consulta. También lo son en temas como catástrofes, salud y protestas. Allí el periodista recurre a fuentes que cuentan en primera persona sus casos como víctimas de un desastre, sus dolencias físicas o sus opiniones en protestas sociales.

Las fuentes del sector empresarial tienen un lugar destacado en las noticias sobre temas económicos, pero esto no se traslada a otros campos en los que el sector también debería ser una voz autorizada para expresarse: energía, transporte y vivienda. La sociedad civil organizada es fuente principal en 


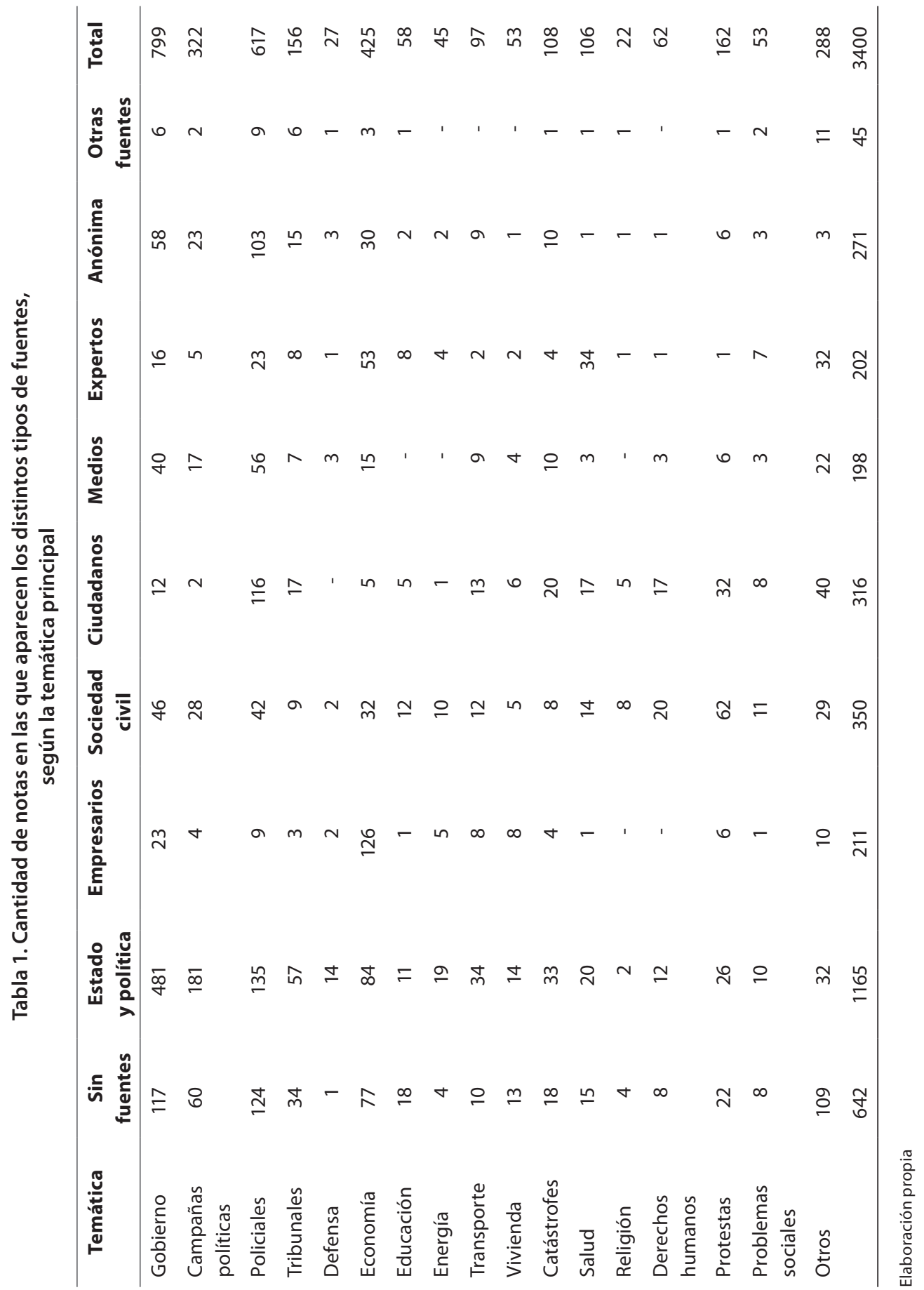


las noticias cuya temática es la protesta social. Además, también es la primera, en menor medida, en noticias sobre temas de gobierno y policiales. Los expertos, especialistas de determinadas ramas del conocimiento, son fuentes esenciales en noticias referidas a economía y salud. Los medios de comunicación, por su parte, son citados como fuente principal en temas policiales, muchas veces por la ausencia de información oficial, como se mencionó anteriormente.

Otra variable para analizar la presencia de las fuentes en las noticias es el medio de comunicación donde dichas noticias se publican. Así, observaremos si se mantiene estable la presencia de las fuentes en los diferentes medios analizados, puesto que estos tienen entre sí diferentes orientaciones ideológicas y de mercado.

En primer lugar, analizaremos la cantidad de fuentes por noticias, según cada medio. Aquí vemos que los porcentajes son similares en todos los medios estudiados. El 75 \% de las noticias analizadas en promedio en los cuatro diarios presentan menos de tres fuentes, que es el piso deseable en una cobertura noticiosa: tener las dos partes involucradas en el tema y una tercera externa que pudiera brindar su mirada sobre el mismo. Esta tendencia se observa, con matices, en todos los medios. Solo La Nación incorpora mayor diversidad de fuentes: el $61 \%$ de las noticias cuenta con menos de tres fuentes. En dicho medio, el porcentaje de noticias que tienen cuatro fuentes duplica al resto de los medios analizados.
En cuanto a la cantidad total de fuentes mencionadas por cada nota, el porcentaje es de 1,25 fuentes por noticia $(\mathrm{f} / \mathrm{n})$. Este porcentaje se mantiene estable en todos los medios analizados, con variaciones no significativas: $1,49 \mathrm{f} / \mathrm{n}$ en La Nación; 1,22 f/n en Página 12; 1,16 f/n en Diario Popular; 1,15 f/n en Clarín. Si partimos del hecho de que a mayor cantidad de fuentes tenemos mayor diversidad de perspectivas sobre el hecho (Rodrigo Alsina, 2003; Mayoral Sánchez, 2005; Amado, 2016b), podemos establecer que estamos todavía lejos del pluralismo pretendido.

Otro dato que notamos es que el $58 \%$ de las noticias de todos los diarios presentan una perspectiva unilateral del tema que tratan, y solo el $23 \%$ muestra diversidad de fuentes o puntos de vista. Esta práctica también es común a todos los diarios, con una leve tendencia a la cobertura unilateral en los diarios Popular y Página 12.

En la tabla 2 se observa la presencia de diferentes tipos de fuentes por cada medio analizado. Lo primero que debemos señalar es que los porcentajes de aparición de cada tipo de fuente se mantienen estables en todos los medios. En todos ellos, más del $30 \%$ de las fuentes citadas pertenecen al Estado o a partidos políticos. En Página 12 y Diario Popular, este porcentaje es mayor, y una posible explicación de este dato puede ser la cercanía ideológica y política entre estos medios y el poder político de turno en los años en que se tomó la muestra: 20122013 (Ruiz, 2014, p. 400). 
Tabla 2. Fuente principal por diarios (porcentaje del total de noticias)

\begin{tabular}{lrcccc}
\hline \multicolumn{1}{c}{ Tipo de fuente } & Clarín & Diario Popular & La Nación & Página 12 & Total general \\
\hline Estado y políticos & 27,59 & 40,96 & 31,92 & 42,67 & 34,26 \\
Sin fuente & 26,30 & 16,14 & 11,97 & 16,13 & 18,88 \\
Sociedad civil & 7,62 & 10,61 & 11,72 & 13,20 & 10,29 \\
Ciudadanos & 9,30 & 10,31 & 9,85 & 7,62 & 9,29 \\
Anónima & 7,94 & 8,97 & 9,98 & 4,69 & 7,97 \\
Empresarios & 6,74 & 2,69 & 9,23 & 5,13 & 6,21 \\
Expertos & 7,30 & 3,29 & 6,61 & 5,28 & 5,94 \\
Medios & 5,85 & 6,13 & 7,61 & 3,37 & 5,82 \\
Otros & 1,36 & 0,90 & 1,12 & 1,91 & 1,32 \\
\hline Total general & 100,00 & 100,00 & 100,00 & 100,00 & 100,00 \\
\hline
\end{tabular}

Elaboración propia

Al analizar la fuente principal de las noticias según cada medio, concluimos que se mantiene la regularidad en la cobertura de todos los diarios, con la excepción de las fuentes del Estado y la política: Diario Popular y Página 12 tienen en más del $40 \%$ de sus noticias al Estado o a partidos políticos como fuente principal.

Otro dato destacable es que en más del $25 \%$ de las noticias de Clarín no hay fuente citada, lo cual podría explicarse, en parte, por la falta de acceso de este medio a las fuentes oficiales, en particular las del poder político de turno. Los diarios más enfrentados al poder político entre 2012 y 2013, Clarín y La Nación, son los que brindan un mayor lugar a las fuentes empresariales, un sector muchas veces opuesto al gobierno nacional argentino (Waisbord, 2013). En los porcentajes de notas cuya fuente principal son miembros y organizaciones de la sociedad civil, Página 12 supera levemente al resto.
Los demás tipos de fuente aparecen prácticamente en los mismos porcentajes en todos los medios.

\section{Conclusiones: la importancia de las fuentes institucionales}

Se observa, en primer lugar, una clara presencia de las fuentes institucionales (son la fuente principal en 6 de cada 10 noticias) con respecto a las fuentes ciudadanas no organizadas (fuente principal en 1 de cada 10 noticias). Esto confirma los resultados de otras investigaciones en las que se analizaron las gestiones de las oficinas de relaciones públicas frente a la iniciativa periodística (Sigal, 1973; De la Torre y Téramo, 2004; Amado, 2016a).

Ya desde fines del siglo pasado se detectó la profesionalización de las oficinas encargadas de la asesoría de prensa (Ramírez, 1995). Como contrapartida de este progreso reciente en lo referente 
a la productividad, encontramos aún serias deficiencias en lo que respecta a la accesibilidad que los asesores de prensa promueven y garantizan a los periodistas. Esto puede observarse en el elevado número de fuentes que no aparecen plenamente identificadas. Si sumamos las noticias que no tienen fuente con aquellas cuya fuente principal es anónima, nos encontramos con que el $27 \%$ de las noticias no cuentan con una fuente identificada. Este dato coincide con el de otras investigaciones (Mayoral Sánchez, 2005; Rotelli, 2010). Periodistas y fuentes trabajan desde lugares diferentes, pero complementarios: ambos forman parte del proceso de construcción de la noticia. Esto se vuelve más claro en la medida en que los clásicos criterios de noticiabilidad (vinculados al suceso en sí, a lo imprevisto, lo que rompe con la rutina) son reemplazados por criterios vinculados al proceso de producción de la noticia: la brevedad, la claridad, la comprensión y la periodicidad (Wolf, 1991).

Otro punto por analizar es el rol del periodismo en el proceso de construcción de las noticias, ya que en casi 3 de cada 10 noticias examinadas no se menciona una fuente informativa o la misma no se encuentra plenamente identificada. Entendemos que quizás una manera de recuperar el vínculo con los lectores sea incluir más voces y perspectivas en las noticias, incluso contradictorias entre sí, a fin de que el público pueda tener más elementos para comprender la compleja realidad actual.

En lo que respecta a la segunda pregunta planteada en este trabajo, podemos decir que la presencia de fuentes se relaciona, en algunos casos, con la temática de la noticia. Esto ocurre, por ejemplo, con los temas de gobierno, en los cuales las fuentes estatales tienen una clara preeminencia sobre los demás actores. Lo mismo ocurre en el caso de temas económicos, en donde actores del sector empresarial son citados como fuentes en un porcentaje mayor que el que tienen en otros temas.

Sin embargo, decimos que esta hipótesis se cumple parcialmente porque las fuentes estatales son las que cuentan con mayor presencia en las noticias, con independencia de la temática que se aborde. Una de cada tres fuentes consultadas por los periodistas proviene del ámbito político, en contraste con el resto de las fuentes, que, en el mejor de los casos, representan una de cada diez consultadas. Además, los temas políticos son prioridad y esto da lugar a una mayor presencia de fuentes estatales, dato que está en consonancia con el fenómeno que Bennett denomina indexing: los periodistas restringen la cantidad y diversidad de fuentes, priorizando las oficiales del sistema político (citado en Castells, 2008). Más aún, Castells (2009) sostiene que las declaraciones de los miembros del poder político reciben una atención especial en las redacciones: "Los medios tienden a clasificar la importancia de un asunto en función de las declaraciones del Gobierno" (p. 219).

En cuanto a la tercera pregunta, se observa que no hay una relación significativa entre la presencia de fuentes y los medios en que se publican las noticias. 
Medios de diferente orientación de mercado, perspectiva ideológica y cercanía al poder político asignan similares porcentajes de aparición a las fuentes estatales y a las no organizadas, por ejemplo. Este dato plantea nuevos desafíos para las investigaciones en periodismo y comunicaciones institucionales: la propiedad de los medios o su línea política o comercial parecen no poder explicar, por sí solos, ciertas prácticas comunes a periodistas que trabajan en diarios diferentes; a su vez, el escenario actual de la producción de información mediática, caracterizado por una cada vez mayor participación de las relaciones públicas, abre una ventana de oportunidad para que organizaciones pertenecientes a diferentes sectores puedan convertirse en una fuente recurrente para los medios de comunicación.

No se hallaron diferencias significativas en el análisis comparativo entre los medios en donde las noticias fueron publicadas. Más allá de la orientación ideológica o el público al que apuntan los diferentes medios analizados, hay regularidades tanto en el número de fuentes por noticia como en los tipos de fuentes consultadas y las temáticas abordadas. Esto nos permitiría hablar de prácticas periodísticas habituales, rutinas de trabajo que se dan en la práctica, con independencia del medio para el que trabajen los profesionales.

El análisis realizado nos permite concluir que existen ciertas prácticas periodísticas presentes en el proceso de construcción de las noticias con relación a las fuentes informativas, que son comunes a periodistas que escriben sobre diferentes temáticas y en distintos medios de comunicación. La ausencia de diversidad de perspectivas y la importancia dada a las fuentes institucionales, en especial a las estatales, son algunas de esas prácticas que se pudieron observar. Esto no implica abrir un juicio de valor sobre las prácticas periodísticas, sino identificar un escenario en el que las instituciones logran instalar su versión en las noticias, en varias ocasiones unilateralmente, mientras que la ciudadanía queda marginada, muchas veces como fuente y otras como un público que debe acostumbrarse a la falta de perspectivas en las noticias.

\section{Referencias}

Amado, A. (2010). Prensa y comunicación. Relaciones informativas responsables. Buenos Aires: La Crujía.

Amado, A. (2014). Los hechos y los dichos en las noticias: la polifonía del discurso periodístico. Revista Olomucensia, 26(2), 1. Recuperado de https://dialnet.unirioja. es/descarga/articulo/5053281.pdf

Amado, A. (2016a). Del newsroom al cloudsroom: el periodista y los productores de información. En L. Lucchessi y L. Videla (Eds.), Desafíos del periodismo en la sociedad del conocimiento (pp. 13-22). Viedma: Universidad Nacional de Río Negro. 
Amado, A. (2016b). La prensa de la prensa: periodismo y relaciones públicas en la información. Buenos Aires: Biblos.

Ambort, M., Loyola, M., Ortiz, A., y Tobar, J. (1996). El periodismo gráfico. Los periodistas, el periódico, los géneros. Córdoba: Op Oloop Ediciones.

Castells, M. (2008). Comunicación, poder y contrapoder en la sociedad red (I). Los medios y la política. Telos, 74, 13-24. Recuperado de https://telos.fundaciontelefonica.com/url-direct/pdf-generator?tipoContenido=articulo\&idConteni do $=2009100116310137$

Castells, M. (2009). Comunicación y poder. Barcelona: Alianza Editorial.

De la Torre, L., y Téramo, M. (2004). La noticia en el espejo. Buenos Aires: Editorial de la Universidad Católica Argentina.

Duarte, J. (Org.). (2011). Assessoria de imprensa e relacionamento com a mídia: teoria e técnica. São Paulo: Atlas.

Gomis, L. (1991). Teoría del periodismo. Cómo se forma el presente. Barcelona: Paidós.

Hanitzsch, T., y Mellado, C. (2011). What Shapes the News around the World? How Journalists in Eighteen Countries Perceive Influences on Their Work. The International Journal of Press/Politics, 20(10), 1-23. https://doi. org/10.1177/1940161211407334

Hellmueller, L., y Mellado, C. (2016). Watchdogs in Chile and the United States: Comparing the Networks of Sources and Journalistic Role Performances. International Journal of Communication, 10(2016), 3261-3280. Recuperado de http:// ijoc.org/index.php/ijoc/article/viewFile/5181/1706

Martini, S. (2000). Periodismo, noticia y noticiabilidad. Bogotá: Norma.

Martini, S., y Luchessi, L. (2004). Los que hacen la noticia. Buenos Aires: Biblos.

Mayoral Sánchez, J. (2005). La batalla de las fuentes. Cuadernos de Periodistas, 5, 13-22.

McCombs, M. (2006). Estableciendo la agenda. El impacto de los medios en la opinión pública y en el conocimiento. Barcelona: Paidós.

McQuail, D. (1998). La acción de los medios. Los medios de comunicación y el interés público. Buenos Aires: Amorrortu.

Mellado, C. (2015). Professional Roles in News Content: Six Dimensions of Journalistic Role Performance. Journalism Studies, 16, 596-614. https://doi.org/10.1080/146167 0X.2014.922276

Mellado, C., Márquez-Ramírez, M., Oller Alonso, M., Mick, J., y Amado, A. (2016). Puesta en práctica de los roles periodísticos: un estudio comparado de Argentina, Brasil, Chile, Ecuador y México. En A. Amado (Ed.), El periodismo por los periodistas (pp. 64-71). Montevideo: Konrad Adenauer; Infociudadana. 
Mellado, C., Márquez-Ramírez, M., Mick, J., Oller Alonso, M., y Olivera, D. (2017). Journalistic Performance in Latin America: A Comparative Study of Professional Roles in News Content. Journalism, 18(9), 1087-1106. https://doi. org/10.1177/1464884916657509

Mellado, C., y Van Dalen, A. (2013). Between Rhetoric and Practice. Explaining the Gap between Role Conception and Performance in Journalism. Journalism Studies. doi: 10.1080/1461670X.2013.838046

Ramírez, T. (1995). La influencia de los gabinetes de prensa: las rutinas periodísticas al servicio del poder. Telos, 40, 47-57. Recuperado de http://www.quadernsdigitals.net/datos/hemeroteca/r_32/nr_453/a_6204/6204.pdf

Riffe, D., Aust, F., y Lacy, S. (1993). The Effectiveness of Random, Consecutive Day, and Constructed Week Sampling in Newspaper Content Analysis. Journalism \& Mass Communication Quarterly, 70(1), 133-139. https://doi. org/10.1177/107769909307000115

Rodrigo Alsina, M. (2003). Confianza en la información mediática. Revista CIDOB d'Afers Internacionals, 61/62, 145-153. Recuperado de http://www.jstor.org/stable/40585964

Rotelli, N. (2010). Comunicación gubernamental: la importancia de la accesibilidad en las relaciones con la prensa. En L. H. Elizalde, D. Fernández Pedemonte y M. Riorda (Ed.), La gestión del disenso. Comunicación gubernamental en problemas. Buenos Aires: La Crujía.

Ruiz, F. (2014). Guerras mediáticas. Buenos Aires: Sudamericana.

Sigal, L. (1973). Reporters and Officials: The Organization and Politics of Newsmaking. Lexington, MA: D. C. Heath and Company.

Waisbord, S. (2013). Vox populista. Medios, periodismo, democracia. Buenos Aires: Gedisa. Wolf, M. (1991). La investigación de la comunicación de masas. Barcelona: Paidós. 